\title{
Stroke unit care, inpatient rehabilitation and early supported discharge
}

\author{
Authors: Helen Rodgers ${ }^{A}$ and Chris Price ${ }^{B}$
}

Stroke units reduce death and disability through the provision of specialist multidisciplinary care for diagnosis, emergency treatments, normalisation of homeostasis, prevention of complications, rehabilitation and secondary prevention. All stroke patients can benefit from provision of high-quality basic medical care and some need high impact specific treatments, such as thrombolysis, that are often time dependent. A standard patient pathway should include assessment of neurological impairment, vascular risk factors, swallowing, fluid balance and nutrition, cognitive function, communication, mood disorders, continence, activities of daily living and rehabilitation goals. Good communication and shared decision making with patients and their families are key to high-quality stroke care. Patients with mild or moderate disability, who are medically stable, can continue rehabilitation at home with early supported discharge teams rather than needing a prolonged stay in hospital. National clinical guidelines and prospective audits are integral to monitoring and developing stroke services in the UK.

\section{Stroke units}

Each year 152,000 people in the UK have a stroke. ${ }^{1}$ Randomised controlled trials and subsequent meta-analyses have clearly shown that care in a multidisciplinary stroke unit reduces death, disability and the need for institutionalisation when compared with general medical wards. ${ }^{2}$ These benefits are seen regardless of the patient's age, gender, stroke type (ie infarct or haemorrhage) or stroke severity (Fig 1). ${ }^{2}$ Based on a number needed to treat of 18 , stroke units prevent death or dependency for 8,400 stroke patients per year. ${ }^{3}$ All acute stroke patients should be treated on a stroke unit (a ward that cares almost exclusively for stroke patients) throughout their inpatient stay unless stroke is not their predominant medical problem. ${ }^{4}$

Author: ${ }^{\text {A }}$ rofessor of stroke care, Newcastle University and honorary consultant stroke physician, Northumbria Healthcare NHS Foundation Trust, Newcastle upon Tyne, UK; ${ }^{B}$ senior lecturer in stroke medicine, Newcastle University and honorary consultant stroke physician, Northumbria Healthcare NHS Foundation Trust, Newcastle upon Tyne, UK
Recently, stroke services in many areas have been reorganised to improve the quality and efficiency of care. ${ }^{5}$ Hyperacute stroke units (HASUs) provide

> rapid specialist assessment and diagnosis on the HASU or in the associated accident and emergency department

$>$ hyperacute treatment, eg thrombolysis

$>$ physiological and neurological monitoring

$>$ early rehabilitation.

Stroke patients usually remain on a HASU for up to 72 hours and are then transferred to an acute stroke unit or stroke rehabilitation unit. Some stroke services have a comprehensive

\section{Key points}

All acute stroke patients should be treated on a stroke unit (a ward that cares almost exclusively for stroke patients) throughout their inpatient stay unless stroke is not the predominant medical problem

Specific emergency treatments are suitable for carefully selected patients (eg thrombolysis and thrombectomy) but provision of high-quality basic medical care is applicable to all patients and is key to improving outcome following acute stroke

Rehabilitation is increasingly based on evidence from clinical trials that should be considered in the context of individual patients' problems and preferences

Early supported discharge should be provided by a single specialist multidisciplinary team to patients with mild/moderate disability; the service should begin within 24 hours of discharge and provide rehabilitation of the same intensity and skill mix as stroke unit care

High-quality research, national and local audits and quality improvement programmes have underpinned the development of evidence-based stroke units and early supported discharge services

KEYWORDS: Cerebrovascular disease, early supported discharge, multidisciplinary care, rehabilitation, stroke unit 


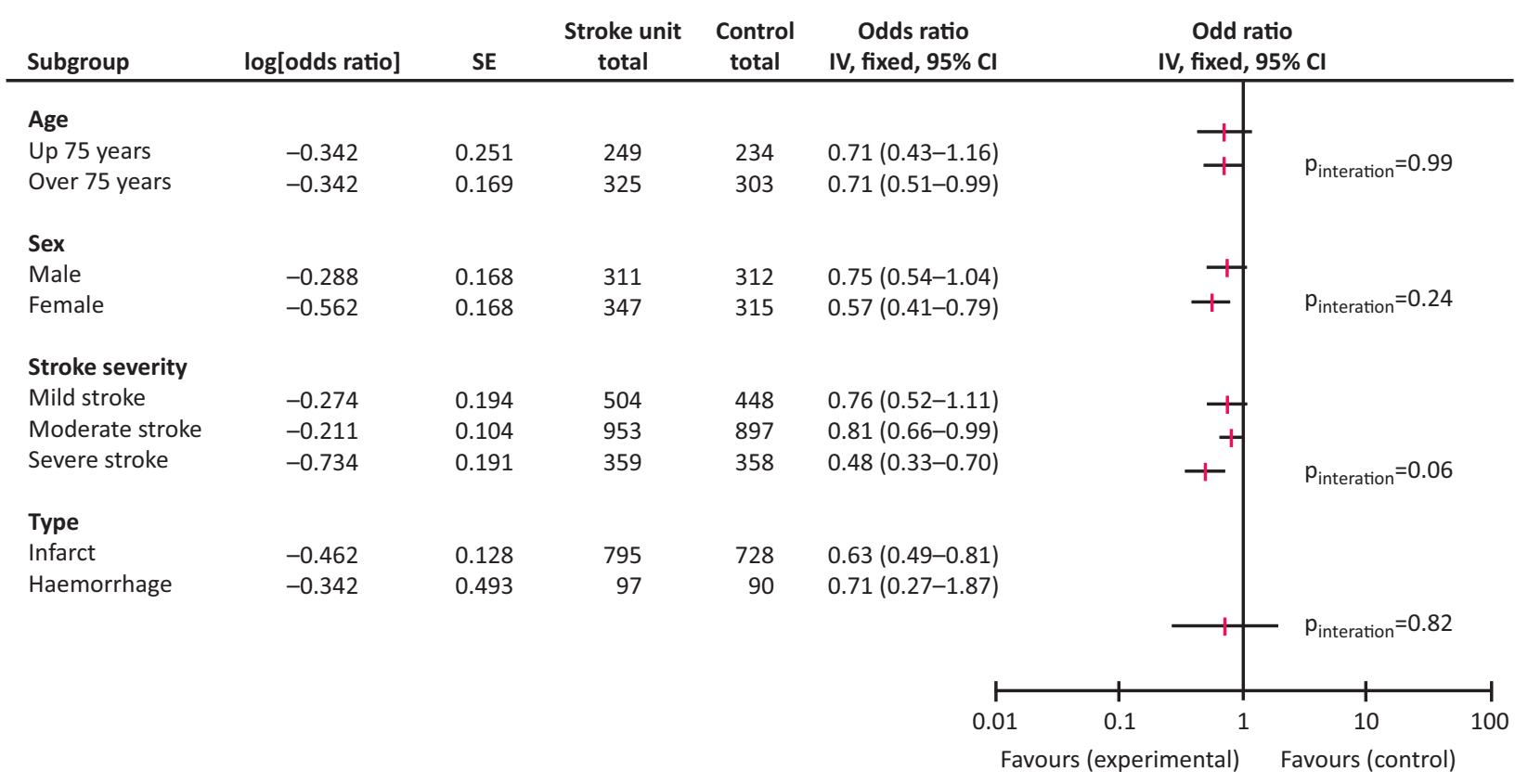

Fig 1. Stroke unit care reduces death and institutionalisation. Reproduced with permission from the Stroke Unit Triallists' Collaboration. ${ }^{2}$

stroke unit that provides hyperacute care, acute care and rehabilitation.

A multidisciplinary stroke unit team consists of doctors, nurses, physiotherapists, occupational therapists, speech and language therapists, clinical/neuropsychologists, dieticians and social workers. ${ }^{4}$ Input may also be provided by pharmacists, orthotics, orthoptists and chiropodists. The stroke unit has close links with radiology, neurosurgery, vascular surgery, neurology, geriatric medicine and cardiology. The multidisciplinary stroke unit team aims to provide personalised holistic care and meets regularly to share updates and plan care. This is usually a weekly multidisciplinary team meeting lasting for 2-3 hours, but many stroke units also have a short 'board round' at least once per day. Patients and their families are actively involved in their care and receive consistent information and support. Stroke unit teams have protocols for common problems and provide a training programme for all team members. ${ }^{3,4}$ They work closely with other components of the stroke service, early supported discharge teams and community services to plan for transfer of care.

\section{Medical care of stroke patients}

Doing the simple things well is fundamental to improving outcome following acute stroke and the importance of providing high-quality basic medical care cannot be over emphasised. National audit data has shown that well organised stroke units that provide timely assessments and treatments have lower mortality rates. ${ }^{6}$ There are highly effective treatments for carefully selected patients, eg thrombolysis, thrombectomy, blood pressure control for primary intracerebral haemorrhage, and decompressive hemicraniectomy for malignant middle cerebral artery infarction. ${ }^{4,7,8}$ These treatments are time dependent and organised stroke care enables clinicians to respond quickly and efficiently. However, improved outcomes were associated with stroke units prior to these interventions being available.

\section{Clinical deterioration}

Patients should be monitored for early and late clinical deterioration. Prompt action to determine a neurological or non-neurological cause (Table 1) and early treatment is essential.

\section{Temperature, blood sugar, oxygen, blood pressure}

Pyrexia early post-stroke can be due to stroke per se or the presence of infection and is associated with a worse outcome. A source of infection should be sought and paracetamol administered as an antipyretic. Hyperglycaemia can occur acutely after stroke and is associated with a poor outcome regardless of diabetic status. ${ }^{9}$ Rigorous glycaemic control has not been shown to improve outcome and current guidelines recommend that blood sugar should be maintained between 5-15 mmol/L. ${ }^{4}$ Routine supplementation of oxygen has not been shown to improve outcome following acute stroke but

Table 1. Common causes of clinical deterioration

following acute stroke

$\begin{array}{ll}\begin{array}{l}\text { Neurological } \\ \text { Haemorrhagic transformation of an } \\ \text { infarct }\end{array} & \begin{array}{l}\text { Non-neurological } \\ \text { Infection (chest or urine) }\end{array} \\ \text { Malignant cerebral oedema } & \text { Pulmonary embolism } \\ \text { Intracerebral haematoma expansion } & \text { Metabolic disturbance } \\ \text { Recurrent cerebral emboli } & \text { Myocardial infarction } \\ \text { Hydrocephalus } & \\ \text { Seizure } & \end{array}$


should be used when patients are hypoxic. Acute blood pressure lowering is only recommended if there is an indication for emergency treatment, eg primary intracerebral haemorrhage (150 mmHg systolic) or thrombolysis (185/110 $\mathrm{mmHg}$ ), and an appropriate protocol for dose titration is available. ${ }^{4}$ Regular use of protocols for the management of abnormal physiological parameters on stroke units has been shown to improve clinical outcomes. ${ }^{10}$

Fluids, swallowing, prophylactic antibiotics, feeding, oral hygiene

As dehydration and malnutrition can easily occur after a stroke, a patient's intake of fluid and food and their nutritional status should be closely monitored. ${ }^{11}$ Dysphagia affects up to $55 \%$ of stroke patients and all patients with suspected stroke should remain nil by mouth and receive intravenous or subcutaneous fluids until their ability to swallow safely has been assessed by a trained nurse soon after admission. ${ }^{12}$ Patients who are screened as being unsafe to swallow should remain nil by mouth and receive non-oral feeding via a nasogastric tube if this is in keeping with the patient's wishes. An early detailed assessment by a speech and language therapist is desirable but nasogastric feeding should not be delayed if this is not available. Videofluoroscopy or fibreoptic endoscopic evaluation of swallowing may be useful for more detailed assessment of patients with persistent dysphagia. Patients with dysphagia beyond 4 weeks or who cannot tolerate a nasogastric tube may require a percutaneous endoscopic gastrostomy. ${ }^{4}$ Pneumonia frequently occurs in patients with dysphagia but two recent randomised controlled trials found that prophylactic antibiotics provided no benefit in terms of prevention of this complication. ${ }^{13,14}$ Maintaining good oral hygiene is important but can be difficult when a patient is unable to clean their teeth. Stroke units should regularly assess a patient's oral hygiene and ensure that staff are trained to provide appropriate oral care. ${ }^{4}$

\section{Prevention of deep-vein thrombosis and pulmonary embolism}

Deep-vein thrombosis (DVT) and pulmonary embolism (PE) are common complications post-stroke. ${ }^{15-18}$ Prophylactic low dose heparin should not be used early after stroke as although this treatment does reduce the risk of DVT and PE, it increases the risk of haemorrhagic transformation of a cerebral infarct. ${ }^{17}$ Graduated pressure stockings are not effective in preventing DVT and PE post-stroke and may precipitate limb ischaemia. ${ }^{16}$ Intermittent pneumatic compression is the treatment of choice as this reduces the incidence of DVT and death post-stroke. ${ }^{15}$

\section{Bladder, bowels and skin}

Urinary incontinence affects up to $40-60 \%$ of acute stroke patients. ${ }^{19}$ The cause of incontinence should be sought, eg pre-existing stress incontinence, urinary tract infection, immobility. Regular toileting can significantly reduce incontinence for patients with cognitive, communication or mobility problems. Urinary catheters should be avoided unless the patient has urinary retention, a sacral pressure sore or if accurate monitoring of fluid balance is essential. Constipation is common after stroke and bowel charts should be used to monitor bowel action with laxatives prescribed, if needed. Pressure sores are avoidable and good skin care, regular turning, pressure-relieving mattresses and specialist seating can reduce their incidence.

\section{Palliative care}

$14 \%$ of acute stroke patients die in hospital and 5\% receive palliative care within 72 hours of admission. ${ }^{4}$ It is important that stroke unit teams have the skills and expertise to provide high-quality care. Palliative care services can provide valuable input for complex cases and support a discharge home.

\section{Rehabilitation}

Rehabilitation aims to maximise a patient's intrinsic and adaptive recovery by addressing specific impairments (eg weakness), activity limitation (eg difficulty walking), restricted social participation (eg less contact with friends) and overall quality of life. Rehabilitation should be personalised, taking into account not only the problems resulting from stroke but also comorbidities and the patient's views and preferences. The potential problems that stroke patients may experience are extensive and listed in the National clinical guideline for stroke (Box 1).

Some problems are very apparent but others are only identified after careful assessment, so screening tools should be routinely used - especially for cognitive and mood disorders. The nature, implications and treatment of problems due to stroke should be explained to patients and their families, who should be regularly updated and involved in decision making. Training should be offered to families, especially for communication and cognitive problems.

Research evidence is unclear about the optimal frequency, intensity and duration of rehabilitation. Greater intensity of practice is generally associated with improved functional outcomes. ${ }^{20-22}$ Current guidelines are pragmatic, recommending that 'people with stroke should accumulate at least 45 minutes of each appropriate therapy every day, at a frequency that enables them to meet their rehabilitation goals, and for as long as they are willing and capable of participating and showing measurable benefit from therapy.' ${ }^{4}$ However, recent research suggests that the timing and intensity of early rehabilitation needs to be carefully considered. Noncompliance and dropout rates are higher if speech and language therapy interventions are commenced early after stroke. ${ }^{23}$ The results of an international multicentre trial, which compared the effectiveness of usual care versus frequent, high-dose mobilisation starting within 24 hours of stroke, showed worst outcomes for the intervention group. ${ }^{24,25}$ At 3 months, $46 \%$ of those in the intervention group had no or minimal disability compared with 50\% in the control group (adjusted odds ratio $0.73,95 \%$ CI $0.59-0.90, p=0.004) .{ }^{25}$ Guidelines now recommend that patients should receive frequent, short, early mobility interventions each day once medically stable. ${ }^{4}$ Therapy should be task specific, progressive, practised frequently and incorporated into the patient's daily routine. ${ }^{4}$

Reduced mobility often dominates early therapy after stroke. Task-specific training may improve sit to stand and functional walking. ${ }^{26}$ Short-term improvements in balance and walking have been found with foot orthosis. ${ }^{27}$ Leg strengthening 
Box 1. Common problems experienced by patients following acute stroke

\author{
Activities of daily living \\ > Personal activities of daily living, eg eating and drinking, \\ washing, dressing \\ > Extended activities of daily living, eg shopping, cooking, \\ housework \\ $>$ Driving \\ $>$ Work and leisure
}

\section{Arm function}

\section{Cognition}

$>$ Cognitive impairment

$>$ Apraxia

$>$ Attention and concentration

$>$ Executive function

$>$ Memory

$>$ Perception

$>$ Spatial awareness

\section{Communication}

> Aphasia

$>$ Dysarthria

$>$ Apraxia of speech

\section{Continence and constipation}

Fatigue

Hydration and nutrition

Mental capacity

Mobility

$>$ Weakness and ataxia

$>$ Balance

Falls and fear of falling

$>$ Walking

\section{Mood and wellbeing}

> Anxiety, depression and psychological distress

$>$ Emotionalism

\section{Mouth care}

Pain

$>$ Musculoskeletal pain

$>$ Shoulder pain and subluxation

$>$ Neuropathic pain (central post-stroke pain)

\section{Sensation}

Sex

\section{Spasticity and contractures}

Swallowing

Vision

Adapted from the National clinical guideline for stroke, 2016.4 exercises, circuit classes and treadmill training (without body weight support) have been shown to be effective. ${ }^{28}$ Loss of arm function affects $69 \%$ of acute stroke patients. ${ }^{29}$ Moderate-quality evidence supports the use of constraint-induced movement therapy, mental practice, mirror therapy, unilateral arm training, virtual reality, high-dose repetitive task training and interventions for sensory impairment, but further research is needed. ${ }^{22}$ Robot-assisted training is also a promising intervention for patients with moderate/severe upper limb weakness. ${ }^{30}$

Aphasia affects nearly one third of stroke patients and can have a major impact on their lives and relationships. Speech and language therapy can improve functional communication, reading comprehension, specific language and writing. ${ }^{23}$ In addition to the cognitive effects of stroke, psychological problems are common and can impede recovery. Brief psychological interventions, eg motivational interviewing or problem-solving therapy, may be helpful. If this is not successful, antidepressant medication may also be needed. Fatigue is a common and under-recognised problem that should be discussed with patients and their families. It is helpful to identify triggers and strategies to manage the problem.

Prior to leaving hospital, patients should be advised about driving according to Driver and Vehicle Licensing Agency regulations. ${ }^{31}$ Patients who cannot return to driving 1 month post-stroke may wish to attend a specialised driving assessment centre. Stroke patients who are under retirement age may benefit from referral to a vocational rehabilitation scheme.

\section{Early supported discharge}

The median length of stay following acute stroke is now 7.3 days (interquartile range $2.8-23.1$ ). ${ }^{32}$ Patients and their families should be fully involved in discharge planning and have a choice about the timing of discharge and the services received. The views and needs of carers should be considered, and appropriate training and support provided. At discharge, patients should be given a named point of contact for information and advice as well as written information about their diagnosis, medication and management plan. ${ }^{4}$

Early supported discharge (ESD) services consist of a single specialist multidisciplinary team that provides rehabilitation for selected patients at the same intensity and skillmix as a stroke unit. ESD is appropriate for patients with mild/moderate disability and should begin within 24 hours of discharge. ${ }^{4}$ Typically, the team consists of therapists, therapy assistants, nurses and support workers visiting a patient from one to four times per day. ESD has been shown to lead to better patient outcomes, increased satisfaction with care and lower NHS costs compared with conventional care. ${ }^{33}$ Prior to discharge, a member of the ESD team may undertake a home visit (with the patient) or an environmental visit (without the patient). The duration of ESD input depends on patient need although some services have a maximum length of input, eg 3 months, following which patients who need further rehabilitation are transferred to other community services.

\section{Conclusion}

Stroke is a common and disabling condition, but many patients benefit from the care delivered by a multidisciplinary specialist stroke service. The evidence for optimising recovery is still 
evolving and should be integrated into the patient care pathway with matching clinical documentation, protocols and staff training.

\section{Conflicts of interest}

The authors have no conflicts of interest to declare.

\section{Acknowledgements}

The authors would like to thank the Intercollegiate Stroke Working Party who prepared the fifth edition of the National clinical guideline for stroke, 2016.

\section{Author contributions}

$\mathrm{HR}$ and $\mathrm{CP}$ wrote the paper.

\section{References}

1 Stroke Association. State of the nation: stroke statistics. London: Stroke Association, 2016

2 Stroke Unit Trialists' Collaboration. Organised inpatient (stroke unit) care for stroke. The Cochrane Database Syst Rev 2013;(9):CD000197.

3 Wellwood I, Langhorne P. Stroke unit care. In: Kalra L, Wolfe C, Rudd A. A practical guide to comprehensive stroke unit care: meeting population needs. Singapore, World Scientific Publishing Co, 2011.

4 Intercollegiate Stroke Working Party. National clinical guideline for stroke, 5th edn. London: Royal College of Physicians, 2016.

5 Ramsay AI, Morris S, Hoffman A et al. Effects of centralizing acute stroke services on stroke care provision in two large metropolitan areas in England. Stroke 2015;46:2244-51.

6 Bray BD, Ayis S, Campbell J et al. Associations between the organisation of stroke services, process of care, and mortality in England: prospective cohort study. BMJ 2013;346:f2827.

7 Law ZK, Appleton J, Bath P, Sprigg N. Management of acute cerebral haemorrhage - an update. Clin Med 2017;166-72.

8 El Tawil S, Muir KW. Thrombolysis and thrombectomy for acute ischaemic stroke. Clin Med 2017;161-5.

9 Weir CJ, Murray GD, Dyker AG, Lees KR. Is hyperglycaemia an independent predictor of poor outcome after acute stroke? Results of a long-term follow up study. BMJ 1997;314:1303-6.

10 Middleton S, McElduff P, Ward J et al. Implementation of evidencebased treatment protocols to manage fever, hyperglycaemia, and swallowing dysfunction in acute stroke (QASC): a cluster randomised controlled trial. Lancet 2011;378:1699-706.

11 Rowat A, Graham C, Dennis M. Dehydration in hospital-admitted stroke patients: detection, frequency, and association. Stroke 2012;43:857-9.

12 Martino R, Foley N, Bhogal S et al. Dysphagia after stroke: incidence, diagnosis, and pulmonary complications. Stroke 2005;36:2756-63.

13 Kalra L, Irshad S, Hodsoll J et al. Prophylactic antibiotics after acute stroke for reducing pneumonia in patients with dysphagia (STROKE-INF): a prospective, cluster-randomised, open-label, masked endpoint, controlled clinical trial. Lancet 2015;386:1835-44.

14 Westendorp WF, Vermeij JD, Zock E et al. The Preventive Antibiotics in Stroke Study (PASS): a pragmatic randomised openlabel masked endpoint clinical trial. Lancet 2015;385:1519-26.

15 CLOTS Trials Collaboration. Effect of intermittent pneumatic compression on disability, living circumstances, quality of life, and hospital costs after stroke: secondary analyses from CLOTS 3, a randomised trial. Lancet Neurology 2014;13:1186-92.

16 CLOTS Trials Collaboration, Dennis M, Sandercock P et al. The effect of graduated compression stockings on long-term outcomes after stroke: the CLOTS trials 1 and 2. Stroke 2013;44:1075-9.
17 Geeganage CM, Sprigg N, Bath MW, Bath PM. Balance of symptomatic pulmonary embolism and symptomatic intracerebral hemorrhage with low-dose anticoagulation in recent ischemic stroke: a systematic review and meta-analysis of randomized controlled trials. J Stroke Cerebrovasc Dis 2013;22:1018-27.

18 Kelly J, Rudd A, Lewis R, Hunt BJ. Venous thromboembolism after acute stroke. Stroke 2001;32:262-7.

19 Thomas LH, Cross S, Barrett J et al. Treatment of urinary incontinence after stroke in adults. Cochrane Database Syst Rev 2008;(1):CD004462.

20 Kwakkel G, van Peppen R, Wagenaar RC et al. Effects of augmented exercise therapy time after stroke: a meta-analysis. Stroke 2004;35:2529-39.

21 Lohse KR, Lang CE, Boyd LA. Is more better? Using metadata to explore dose-response relationships in stroke rehabilitation. Stroke 2014;45:2053-8.

22 Pollock A, Farmer SE, Brady MC et al. Interventions for improving upper limb function after stroke. Cochrane Database Syst Rev 2014;(11):CD010820.

23 Brady MC, Kelly H, Godwin J, Enderby P, Campbell P. Speech and language therapy for aphasia following stroke. Cochrane Database Syst Rev 2016; (6):CD000425.

24 Bernhardt J, Churilov L, Ellery F et al. Prespecified dose-response analysis for A Very Early Rehabilitation Trial (AVERT). Neurology 2016;86:2138-45.

25 The AVERT Trial Collaboration group. Efficacy and safety of very early mobilisation within $24 \mathrm{~h}$ of stroke onset (AVERT): a randomised controlled trial. Lancet 2015;386:46-55.

26 Pollock A, Baer G, Campbell P et al. Physical rehabilitation approaches for the recovery of function and mobility following stroke. Cochrane Database Syst Rev 2014;(4):CD001920.

27 Tyson SF, Kent RM. Effects of an ankle-foot orthosis on balance and walking after stroke: a systematic review and pooled metaanalysis. Arch Phys Med Rehabil 2013;94:1377-85.

28 Veerbeek JM, van Wegen E, van Peppen R et al. What is the evidence for physical therapy poststroke? A systematic review and meta-analysis. PloS ONE 2014;9:e87987.

29 Nakayama H, Jorgensen HS, Raaschou HO, Olsen TS. Recovery of upper extremity function in stroke patients: the Copenhagen Stroke Study. Arch Phys Med Rehabil 1994;75:394-8.

30 Mehrholz J, Pohl M, Platz T, Kugler J, Elsner B. Electromechanical and robot-assisted arm training for improving activities of daily living, arm function, and arm muscle strength after stroke. Cochrane Database Syst Rev 2015;(11):CD006876.

31 Driver and Vehicle Licensing Agency. Neurological disorders: assessing fitness to drive. Stroke and Transient Ischaemic Attack (TIA) - including amaurosis fugax. Swansea: DVLA, 2016. https://www.gov.uk/guidance/ neurological-disorders-assessing-fitness-to-drive\#stroke-andtransientischaemic-attack-tia\%E2\%80\%93including-amaurosis-fugax [Accessed 20 January 2017].

32 Royal College of Physicians, Clinical Effectiveness and Evaluation Unit on behalf of the Intercollegiate Stroke Working Party. Sentinel Stroke National Audit Programme (SSNAP) clinical audit JanuaryMarch 2016 public report. London: RCP, 2016.

33 Fearon P, Langhorne P, Early Supported Discharge Trialists. Services for reducing duration of hospital care for acute stroke patients. Cochrane Database Syst Rev 2012;(9):CD000443.

Address for correspondence: Professor Helen Rodgers, Stroke Research Group, Institute of Neuroscience, Newcastle University, 3-4 Claremont Terrace, Newcastle upon Tyne NE2 4AE, UK.

Email: helen.rodgers@newcastle.ac.uk 